Biochimica et Biophysica Acta, 478 (1977) 316-330

(C) Elsevier/North-Holland Biomedical Press

BBA 99008

\title{
THE 73 S RIBOSOME OF NEUROSPORA CRASSA IS THE NATIVE MITOCHONDRIAL RIBOSOME
}

\author{
R. MICHEL, G. HALLERMAYER, M.A. HARMEY *, F. MILLER and W. NEUPERT
}

Institut für Physiologische Chemie, Physikalische Biochemie und Zellbiologie der Universität München, 8000 München, Goethestr. 33 (G.F.R.)

(Received January 19th, 1977)

\section{Summary}

The effect of the inclusion of EDTA and of heparin, in media used in the isolation of mitochondria, on the mitochindrial ribosome has been investigated.

1. Mitochondria isolated from Neurospora crassa in the presence of EDTA contain only a single type of monomeric ribosome, viz. $73 \mathrm{~S}$.

2. Mitochondria isolated in the presence of $\mathrm{Mg}^{2+}$ contain both 79-S and 73-S monomeric ribosomes. The heterogeneity of the ribosomes was demonstrated by (a) ultracentrifugation on sucrose gradients, (b) electron microscopy, (c) immunoprecipitation with antibodies against mitochondrial 73-S and 79-S cytoplasmic ribosomes, (d) gel electrophoresis of high and low molecular weight RNAs.

3. Inclusion of heparin in all media used for the isolation of mitochondria and ribosomes resulted in (a) dissociation of 73-S mitochondrial ribosomes into 50-S and 37-S subunits; (b) stabilization of 79-S cytoplasmic ribosomes; (c) in the case of mitochondria isolated in the presence of $\mathrm{Mg}^{2+}$ containing both 73-S and 79-S ribosomes, heparin causes the selective dissociation of the $73 \mathrm{~S}$ monosome to yield ribosomes containing only a single monomeric ribosome type, viz. $79 \mathrm{~S}$.

4. It is concluded that (a) the 79-S ribosomes present in mitochondria isolated in the presence of $\mathrm{Mg}^{2+}$ are contaminating cytoplasmic ribosomes, (b) the 73-S ribosomes are the real functional mitochondrial ribosomes of Neurospora crassa.

\section{Introduction}

Controversy still surrounds the nature of the mitochondrial ribosome of Neurospora crassa. Recent reviews have pointed out that it is very difficult at

* Present address: Department of Botany, University College Dublin, Irish Republic. 
present to draw final conclusions on the character of the functional mitochondrial ribosome [1-3]. For a long time the $73 \mathrm{~S}$ ribosome as first described by Küntzel and Noll [4] was accepted as the functional mitochondrial ribosome. Dissociation yielded subunits with $S$ values of $50 \mathrm{~S}$ and $37 \mathrm{~S}$. Doubts on the validity of this belief have been raised in recent papers and reviews [1,2,5-7]. These doubts stem principially from the initial observations by Datema et al. [5], who have observed a mitochondrial ribosome from $N$. crassa with a sedimentation coefficient of $80 \mathrm{~S}$. The authors have suggested that the $73 \mathrm{~S}$ ribosome results from the presence of EDTA in the media used in the isolation of mitochondria. It was claimed that EDTA removes $5 \mathrm{~S}$ RNA and a number of proteins thus altering the sedimentation behaviour of the ribosome. Furthermore, it was stated that inclusion of $\mathrm{Mg}^{2+}$ in the isolation media for mitochondria prevents this loss of RNA and protein.

The views expressed by these workers have far reaching implications for much of the work on mitochondrial ribosomes, as it is fairly standard practice to wash mitochondrial preparations with EDTA-containing media. To date no systematic study of the possible damaging effects of isolation in the presence of EDTA on mitochondrial ribosomes has appeared.

We present below a comparison of the physical and immunological properties of ribosomes obtained from mitochondria isolated in the presence and absence of $\mathrm{Mg}^{2+}$. We furthermore have studied the effects of heparin on mitochondrial and cytoplasmic ribosomes since it has been claimed that only in the presence of this agent can a homogeneous population of mitochondrial ribosomes be obtained [5].

\section{Materials and Methods}

Growth conditions. Neurospora hyphae, wild-type 74A re, were grown in aerated liquid cultures at $25^{\circ} \mathrm{C}$ in Vogel's minimal medium [8] supplemented with $2 \%$ sucrose. The inoculum was $5 \cdot 10^{5}$ conidia per $\mathrm{ml}$. The cells were harvested in the mid-log phase $(18 \mathrm{~h})$ by filtration.

Preparation of mitochondria. Mitochondria were isolated from 18-h cultures. Hyphae were suspended in 10 volumes of isolation medium in a bottom driven macerator and the suspension was passed through a tissue mill as described by Weiss et al. [9]. The homogenate was centrifuged $10 \mathrm{~min}$ at $1500 \times \mathrm{g}$ to sediment cell walls and debris. Mitochondria were sedimented by centrifugation at $17000 \times g$ for $15 \mathrm{~min}$. The pellet contained crude mitochondria which were washed by resuspension and centrifugation at $17000 \times \mathrm{g}$ for $15 \mathrm{~min}$ as described in Table I.

TABLE I

ISOLATION PROCEDURES OF MITOCHONDRIA

\begin{tabular}{lllll}
\hline Designation of mitochondrial preparations & $\mathrm{SE}_{1.0} \mathrm{~T}$ & $\mathrm{SAMT}$ & $\mathrm{SME}$ & $\mathrm{SE}_{0.1} \mathrm{~T}$ \\
Isolation medium & $\mathrm{A}$ & $\mathrm{B}$ & $\mathrm{B}$ & $\mathrm{C}$ \\
Wash medium & $2 \times \mathrm{A}$ & $3 \times \mathrm{B}$ & $2 \times \mathrm{A}$ & $2 \times \mathrm{C}$ \\
\hline
\end{tabular}

A: $0.44 \mathrm{M}$ sucrose, $1 \mathrm{mM}$ EDTA, $10 \mathrm{mM}$ Tris $\cdot \mathrm{HCl}$ (pH 7.6)

B : $0.44 \mathrm{M}$ sucrose, $0.1 \mathrm{M} \mathrm{NH}_{4} \mathrm{Cl}, 10 \mathrm{mM} \mathrm{MgCl}_{2}, 10 \mathrm{mM}$ Tris $\cdot \mathrm{HCl}$ (pH 7.6)

C : $0.44 \mathrm{M}$ sucrose, $0.1 \mathrm{mM}$ EDT A, $10 \mathrm{mM}$ Tris $\cdot \mathrm{HCl}(\mathrm{pH} \mathrm{7.6)}$ 
Isolation of ribosomes and gradient centrifugation. Mitochondrial ribosomes were prepared by lysis of mitochondria in $0.44 \mathrm{M}$ sucrose, $0.1 \mathrm{M} \mathrm{NH}_{4} \mathrm{Cl}$, $0.01 \mathrm{M} \mathrm{MgCl}_{2}, 0.01 \mathrm{M}$ Tris $\cdot \mathrm{HCl}(\mathrm{pH} 7.5)$ containing $1 \%$ Triton X-100. After a clarifying spin $(15 \mathrm{~min}, 27000 \times \mathrm{g})$ the lysate was placed on a $1.5 \mathrm{ml}$ cushion of $1.4 \mathrm{M}$ sucrose in $0.1 \mathrm{M} \mathrm{NH}_{4} \mathrm{Cl}, 0.01 \mathrm{M} \mathrm{MgCl}_{2}, 0.01 \mathrm{M}$ Tris $\cdot \mathrm{HCl}(\mathrm{pH} 7.5$ ), and centrifuged for $2 \mathrm{~h}$ at $144000 \times \mathrm{g}$. The resulting ribosomal pellet was resuspended in $0.1 \mathrm{M} \mathrm{NH}{ }_{4} \mathrm{Cl}, 0.01 \mathrm{M} \mathrm{MgCl}_{2}, 0.01 \mathrm{M}$ Tris $\cdot \mathrm{HCl}$, ( $\left.\mathrm{pH} 7.5\right)$, and the suspension layered onto a sucrose gradient. Gradient density centrifugation was carried out as described previously [10] and the gradients were monitored with a Zeiss PMQ II photometer, using a flow cell ( $50 \mu \mathrm{l}, 1 \mathrm{~cm}$ path-length).

Cytoplasmic ribosomes were prepared in a buffer containing $0.44 \mathrm{M}$ sucrose, $0.1 \mathrm{M} \mathrm{NH}_{4} \mathrm{Cl}, 0.01 \mathrm{M} \mathrm{MgCl}_{2}, 0.01 \mathrm{M}$ Tris $\cdot \mathrm{HCl}$ (ph 7.5). Cells were disrupted in a tissue mill [9] and the homogenate was centrifuged for $20 \mathrm{~min}$ at $17000 \times \mathrm{g}$. To the resulting supernatant Triton $\mathrm{X}-100$ was added to a final concentration of $1 \%$. After a clarifying spin ( $15 \mathrm{~min}, 27000 \times \mathrm{g})$ the lysate was centrifuged as described above. Gradients were calibrated by the inclusion of ${ }^{3} \mathrm{H}$-labeled Escherichia coli ribosomes.

Electron microscopy of mitochondria. Mitochondria were prepared in the absence $\left(\mathrm{SE}_{1.0} \mathrm{~T}\right.$ : $0.44 \mathrm{M}$ sucrose, $1.0 \mathrm{mM}$ EDTA, $10 \mathrm{mM}$ Tris $\cdot \mathrm{HCl}(\mathrm{pH} 7.5)$ ) and in the presence of $\mathrm{Mg}^{2+}$ (SAMT: $0.44 \mathrm{M}$ sucrose, $0.1 \mathrm{M} \mathrm{NH}_{4} \mathrm{Cl}, 10 \mathrm{mM}$ $\mathrm{MgCl}_{2}, 10 \mathrm{mM}$ Tris $\cdot \mathrm{HCl}(\mathrm{pH} 7.6)$ ). Both preparations were treated with antibodies specific for cytoplasmic ribosomes as described previously [11]. Mitochondrial pellets were fixed in $1 \% \mathrm{OsO}_{4}$ in $0.1 \mathrm{M}$ cacodylate buffer ( $\mathrm{pH} 7.2$ ) dehydrated in ethanol and embedded in Epon. Thin sections were cut on a LKB ultratome III, stained with uranyl acetate and lead citrate and viewed in a Siemens Elmiskop 102.

Immunoprecipitation on cytoplasmic and mitochondrial ribosomes. Antibodies against cytoplasmic and mitochondrial ribosomes were prepared as reported previously [11]. Quantitative immunoprecipitation of mitochondrial and cytoplasmic ribosomes from gradient fractions was carried out in the presence of $1 \%$ Triton $\mathrm{X}-100$. The immunoprecipitate reaction mixtures were incubated at $4^{\circ} \mathrm{C}$ for $15 \mathrm{~h}$ to allow precipitation.

Preparation of mitochondrial RNA and gel electrophoresis. Total nucleic acid was extracted from mitochondria as described by Leaver and Ingle [12] and precipitated with ethanol. Polyacrylamide gel electrophoresis of the mitochondrial RNA was carried out by the method of Loening [13]. Fractionation of high molecular weight RNA species was carried out on $2.4 \%$ polyacrylamide gels for $2.5 \mathrm{~h}$ at $50 \mathrm{~V}(6 \mathrm{~mA} / 8 \mathrm{~cm}$ gel $)$ and then scanned in a Gilford spectrophotometer. All gels were pre-run before loading for 30 min under the conditions outlined. The molecular weights were determined by coelectrophoresis of $E$. coli ribosomal RNA of molecular weights $1.1 \cdot 10^{6}$ and $0.56 \cdot 10^{6}$ as standards. Low molecular weight RNA components were separated on $7.5 \%$ gels using the same electrophoretic conditions as for the high molecular weight RNA.

Isolation of mitochondrial and cytoplasmic ribosomes in the presence of heparin. Heparin (sodium salt, grade II, Sigma Chemical Co., St. Louis, Mo., U.S.A.) was added at a concentration of $0.5 \mathrm{mg} / \mathrm{ml}$ to all media used in the isolation of crude ribosomes in experiments on the effects of heparin on the sedimentation behaviour of the ribosomes. 


\section{Results and Discussion}

Ultracentrifugation of ribosomes from mitochondria isolated in the presence and absence of $\mathrm{Mg}^{2+}$

Mitochondria were isolated in the presence and absence of $\mathrm{Mg}^{2+}$ as outlined in Table I. The mitochondria obtained by these different isolation procedures were lysed in the presence of $\mathrm{Mg}^{2+}$ (SAMT buffer: $0.44 \mathrm{M}$ sucrose, $0.1 \mathrm{M}$ $\mathrm{NH}_{4} \mathrm{Cl}, 0.01 \mathrm{M} \mathrm{MgCl}_{2}, 0.01 \mathrm{M}$ Tris $\cdot \mathrm{HCl}(\mathrm{pH} \mathrm{7.5))}$. Ribosomes were isolated by ultracentrifugation and analysed by sucrose density gradient centrifugation. The sedimentation profiles obtained using the different procedures (cf. Table I) are shown in Fig. 1.

When mitochondria were isolated in the presence of $1.0 \mathrm{mM}$ EDTA (Fig. 1A) or $0.1 \mathrm{mM}$ EDTA (Fig. 1D), the ribosomes show a prominent $73 \mathrm{~S}$ peak. $50-\mathrm{S}$ and 37-S subunits are also present and a small amount of dimers and trimers.

The ribosomal gradients obtained when both mitochondria and ribosomes were isolated in the presence of $\mathrm{Mg}^{2+}$ showed a major ribosomal component with a sedimentation coefficient of $79 \mathrm{~S}$ (Fig. 1B). The $79 \mathrm{~S}$ peak however, had a shoulder corresponding to a component with an $S$ value of $73 \mathrm{~S}$. The gradient also showed the presence of two types of dimers; the $103 \mathrm{~S}$ dimer belongs to the $73 \mathrm{~S}$ ribosome [4], the $109 \mathrm{~S}$ dimer has the same sedimentation velocity as the cytoplasmic dimer. A small peak was also present which corresponds to the cytoplasmic trimers.

When mitochondria were isolated in the presence of $\mathrm{Mg}^{2+}$ but subsequently washed with buffer containing $0.1 \mathrm{mM}$ EDTA (SME, cf. Table I) (Fig. 1C), the same components were present in the gradients. The relative amounts were different, the 50-S and 37-S subunits accounting for a much higher proportion of total ribosomes.

The yield of ribosomes from mitochondria isolated in the presence of $\mathrm{Mg}^{2+}$ was considerably higher than from corresponding mitochondria isolated in the presence of EDTA. Average yield of ribosomes were $1.5-2.5 A_{260 \mathrm{~nm}} / \mathrm{mg}$ mitochondrial protein for SAMT-mitochondria compared to $0.5-0.8 A_{260 \mathrm{~nm}} / \mathrm{mg}$ mitochondrial protein for SET-mitochondria.

These findings are largely in agreement with those of Datema et al. [5]. We differ from these authors in their interpretation. We explain these observations that Neurospora mitochondria isolated in the presence of $\mathrm{Mg}^{2+}$ are contaminated by 79-S cytoplasmic ribosomes. The contamination gives rise to heterogeneous gradients and very high yield of ribosomes. In other organisms inclusion of $\mathrm{Mg}^{2+}$ in the isolation media used to prepare mitochondria has been described to cause extensive aggregation of cytoplasmic ribosomes, their aggregation of cytoplasmic ribosomes, their aggregation with cellular membranes and binding to the outer mitochondrial membrane [14-16]. On the other hand, mitochondria isolated in the presence of EDTA, are essentially free from cytoplasmic ribosomes. The $73 \mathrm{~S}$ monosome, therefore represents the mitochondrial ribosome freed of contaminating cytoplasmic ribosomes.

The reader may find it difficult not to be confused by the different $S$ values determined for the ribosomes discussed here. Whereas there is no controversy about the ribosomes from EDTA isolated mitochondria to have $73 \mathrm{~S}$, the cytoplasmic ribosomes were differently regarded to have $S$ values between $77 \mathrm{~S}$ and 


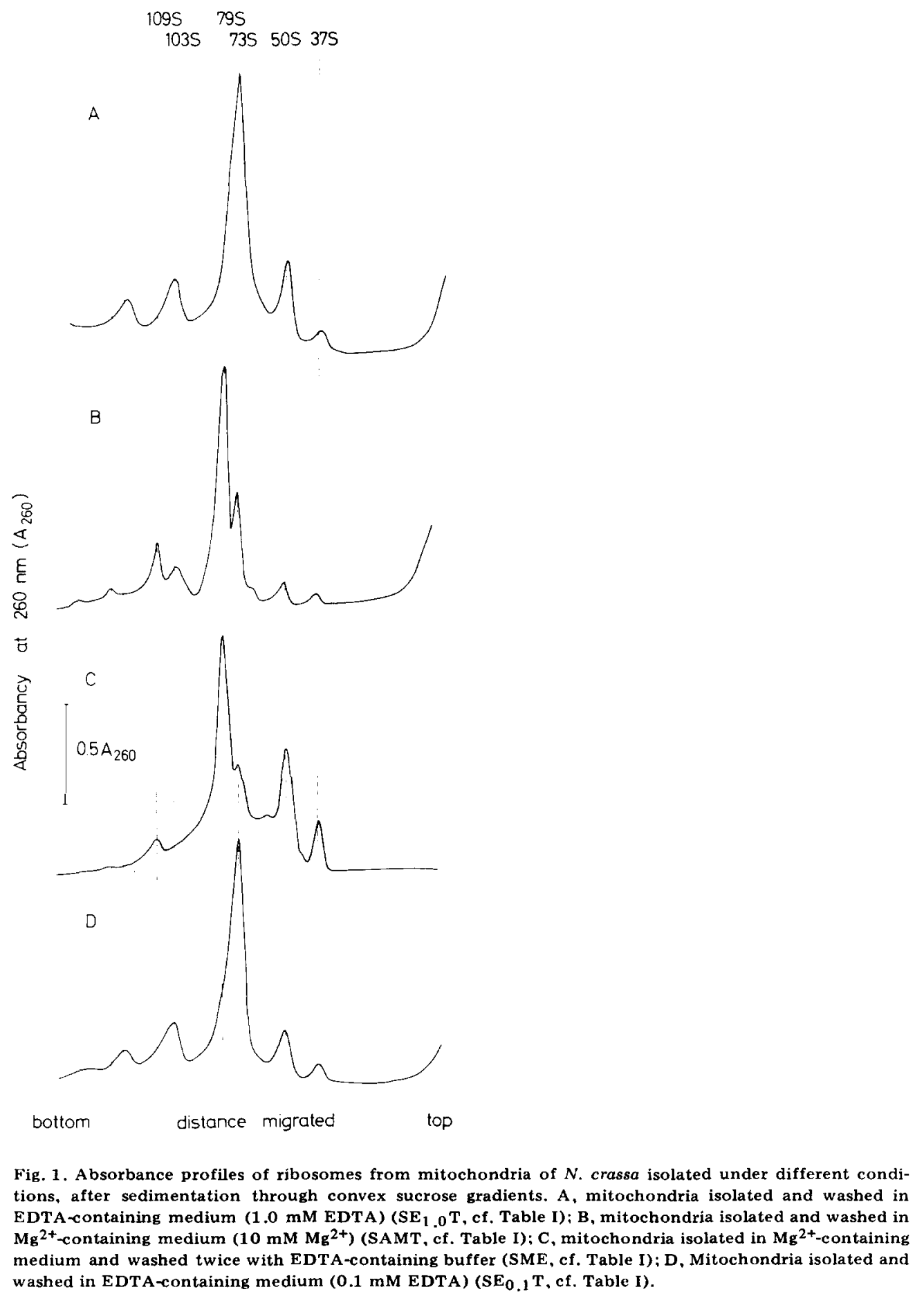


$83.5 \mathrm{~S}[4,17,23]$. The variation may result from differences in experimental conditions and from the use of different methods in calibrating gradients. On this basis we found it not practicable to distinguish a possible $80 \mathrm{~S}$ ribosome from cytoplasmic ribosomes by parallel centrifugation of ribosome samples. A clear resolution even by cocentrifugation to within 1-3 $\mathrm{S}$ units is very difficult to achieve with the techniques applied in these studies.

It may be significant in this context that in earlier reports of Datema et al. [5] the subunits of both 80-S and 73-S ribosomes were presented as being $52 \mathrm{~S}$ and $39 \mathrm{~S}$. Recently however, they ascribe values of 55 and 40 to the subunits of the $80 \mathrm{~S}$ ribosome and 50 and 40 to the subunits of the $73 \mathrm{~S}$ ribosome [7]. These different $S$ values may reflect the difficulties of accurately calibrating gradients.

\section{Electron microscopic examination of mitochondrial preparations}

The presence of cytoplasmic ribosomes in mitochondria isolated in the presence of $\mathrm{Mg}^{2+}$ was further demonstrated by electron microscopic examination. $\mathrm{SE}_{1.0} \mathrm{~T}$-isolated mitochondria and SAMT-isolated mitochondria were treated with specific antibodies against cytoplasmic ribosomes. Electron micrographs of both preparations are shown in Fig. 2 . In $\mathrm{SE}_{1.0} \mathrm{~T}$-isolated preparations contaminating cytoplasmic ribosomes are not evident (Fig. 2A). The SAMTisolated mitochondria (Fig. 2B), however, show the presence of contaminating ribosomal material as well as non-identified contaminants. The antibodies cause extensive clumping of the external ribosomes in SAMT-isolated preparations. These observations indicate quite clearly that the SAMT-isolated mitochondria contain a complement of contaminating ribosomes, which are absent in EDTAwashed mitochondria.

The electron microscopic findings support the view that the heterogeneous profiles shown in Fig. 1 are due to the cosedimentation of cytoplasmic and mitochondrial ribosomes.

\section{Cosedimentation and immunoprecipitation experiments}

The presence of cytoplasmic and mitochondrial ribosomes in the 73-79 S peak fraction of ribosomes obtained from SAMT-isolated mitochondria was demonstrated by immunoprecipitation of cytoplasmic and mitochondrial ribosomes and by mixing experiments.

A culture was grown in the presence of $\left[{ }^{3} \mathrm{H}\right]$ leucine, mitochondria were isolated with SAMT medium and mitochondrial ribosomes were isolated. These ribosomes were mixed with cytoplasmic ribosomes prepared from the postmitochondrial supernatant, of cells grown in the presence of $\left[{ }^{14} \mathrm{C}\right]$ leucine. The mixture was subjected to gradient centrifugation and ${ }^{3} \mathrm{H}$ and ${ }^{14} \mathrm{C}$ radioactivity measured in the gradient fractions (Fig. 3). It can be seen that the main peak of the SAMT-isolated ribosomes coincides exactly with the monomer peak of the cytoplasmic ribosomes. As in the gradient profiles of SAMT-isolated ribosomes (cf. Fig. 1B) a shoulder at $73 \mathrm{~S}$ and a peak at about $50 \mathrm{~S}$ can be seen.

To resolve the components of the gradients shown in Fig. 1B we prepared ${ }^{3} \mathrm{H}$-labeled SAMT-isolated ribosomes and fractionated the gradients. The fractions were halved and treated, respectively, with antibodies to cytoplasmic ribosomes and with antibodies to ribosomes from SET-isolated mitochondria. 

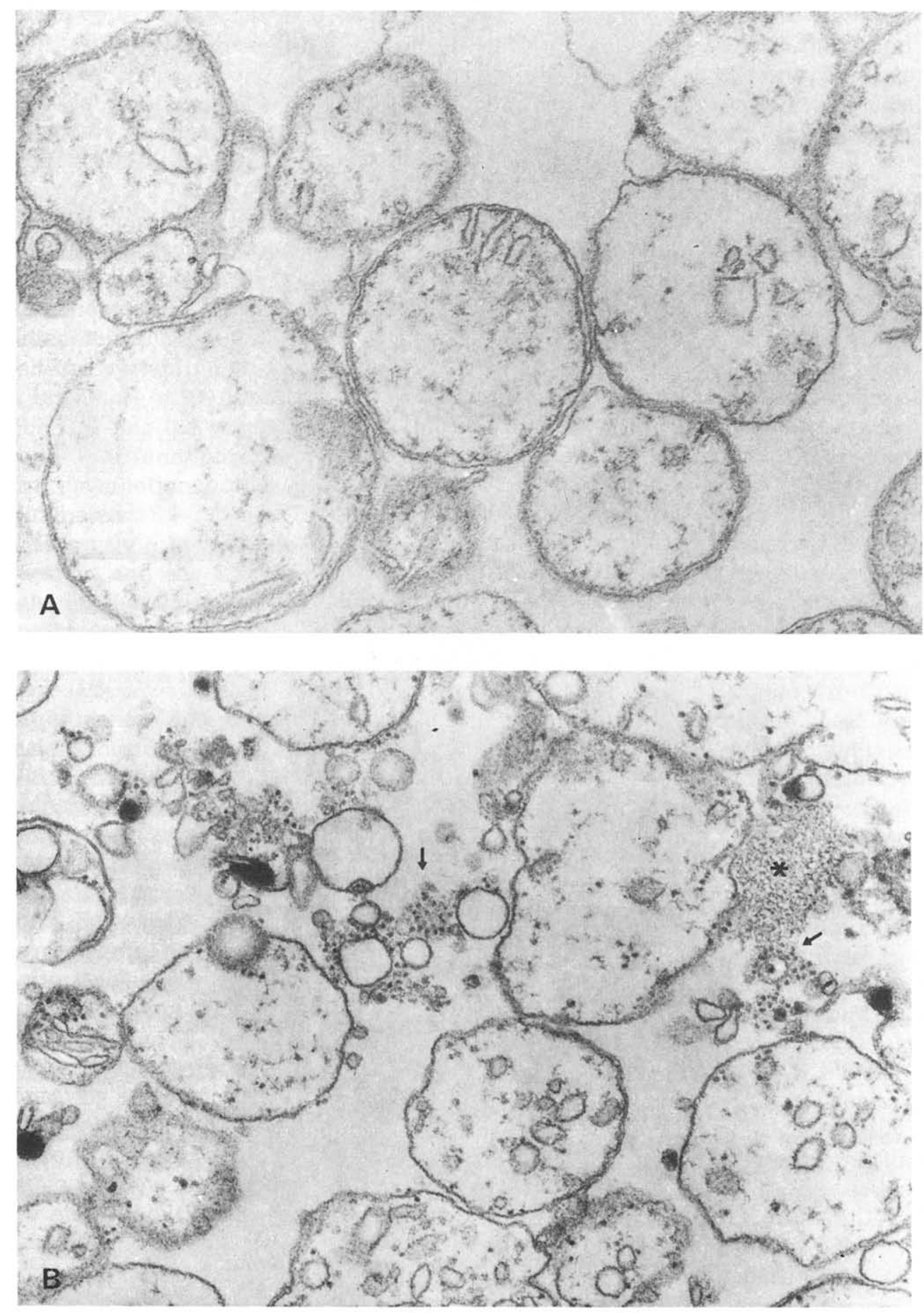

Fig. 2. Electron micrographs of mitochondria isolated in $\mathrm{SE}_{1,0} \mathrm{~T}$ (A) and $\mathrm{SAMT}$ (B). Suspensions in $\mathrm{SE}_{1,0} \mathrm{~T}$ and $\mathrm{SAMT}$ were treated with antibodies to cytoplasmic ribosomes for $1 \mathrm{~h}$ and resedimented. Mitochondria in both preparations are swollen. In the SAMT-preparations clusters of ribosomes are marked by arrows. Area marked by asterisk may represent chromatin. Magnification: 44000. 

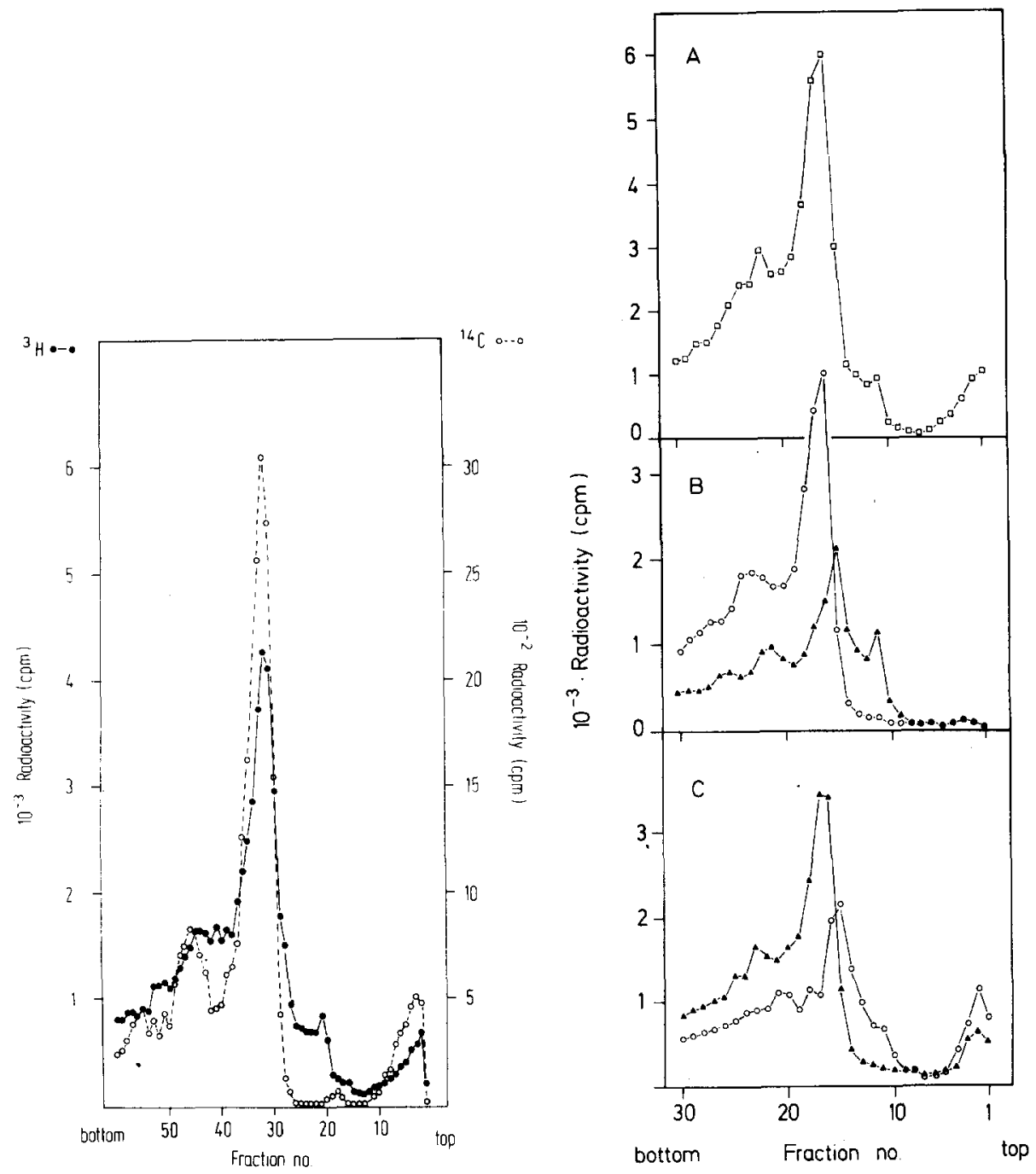

Fig. 3. Distribution of radioactivity in a sucrose density gradient after cocentrifugation of ${ }^{3} \mathrm{H}$-labeled ribosomes from SAMT-isolated mitochondria and ${ }^{14} \mathrm{C}$-labeled cytoplasmic ribosomes. $\bullet-\bullet$, ribosomes from SAMT-isolated mitochondria $\left({ }^{3} \mathrm{H}\right.$-labeled); -0 , cy toplasmic ribosomes $\left({ }^{14} \mathrm{C}\right.$-labeled).

Fig. 4. Immunoprecipitation of ${ }^{3} \mathrm{H}$-labeled ribosomes isolated from mitochondria prepared in the presence of $\mathrm{Mg}^{2+}$ (SAMT) after density gradient centrifugation. (A) Total radioactivity in the gradient fractions. (B) Radioactivity in the immunoprecipitates. (C) Radioactivity in the supernatant of the immunoprecipitations. $\bigcirc-0$, antiserum against cytoplasmic ribosomes: $\longrightarrow$, antiserum against mitochondrial 73-S ribosomes.

The distribution of the total radioactivity in the gradient fractions is shown in Fig. 4A, the distribution of the radioactivity immunoprecipitated in Fig. $4 \mathrm{~B}$. The antibodies against cytoplasmic ribosomes precipitate mainly 79-80-S ribosomes and 109-S dimers. The immunoprecipitates obtained with antibodies against mitochondrial 73-S ribosomes show a quite different profile, namely a main peak at $73 \mathrm{~S}$ and two smaller ones corresponding to about $50 \mathrm{~S}$ and 
$103 \mathrm{~S}$. The antibodies against 73-S mitochondrial ribosomes precipitate about $35 \%$ of total radioactivity present in the gradient. A converse pattern for the distribution of the radioactivity is shown in the supernatant fractions (Fig. 4C).

The SAMT-isolated ribosomes therefore contain 73-S ribosomes which are recognized by the antibody to ribosomes obtained from $\mathrm{SE}_{1,0} \mathrm{~T}$-isolated mitochondria. This is significant in view of the fact that ribosomes from SAMTisolated mitochondria had at no time been exposed to EDTA. Ribosomes from SAMT-isolated mitochondria further contain 79-S ribosomes which react with antibody to cytoplasmic ribosomes, but not with antibody to ribosomes from $\mathrm{SE}_{1,0} \mathrm{~T}$-isolated mitochondria. Clearly, the 79-S ribosomes in the ribosomes from SAMT-isolated mitochondria are immunologically identifiable as cytoplasmic ribosomes. It is apparent from these data that mitochondria isolated in the presence of $\mathrm{Mg}^{2+}$ contain cytoplasmic ribosomes. The precipitation of radioactivity furthermore shows that the cytoplasmic ribosomes occur in quantities sufficient to obscure the appearance of 73-S mitochondrial ribosomes in absorbance profiles.

Van den Bogert and de Vries have compared electrophoretic patterns of ribosomal proteins from mitochondria isolated in the presence of $\mathrm{Mg}^{2+}$ and EDTA [6]. Ribosomes prepared in the presence of heparin from mitochondria which were isolated in $\mathrm{Mg}^{2+}$-containing media $(80 \mathrm{~S})$ contained some 69 proteins, while ribosomes prepared from EDTA-isolated mitochondria in heparin-free buffer $(73 \mathrm{~S})$ had only 61 proteins. Comparison of the patterns from the two types of mitochondrial ribosomes showed that 44 spots were in identical or similar positions (23 in the large and 21 in the small subunit) suggesting partial identity. The $\mathrm{Mg}^{2+}$-plus heparin-isolated ribosomes contained a further 25 proteins which were found only in the $80 \mathrm{~S}$ ribosome, while the ribosomes from EDTA-prepared mitochondria contained 17 unique proteins. The protein patterns obtained with cytoplasmic ribosomes were different from those obtained with either types of ribosomes from mitochondria. This finding was used to assess the extent of cytoplasmic contamination of mitochondrial ribosomes. The authors report that the contamination by cytoplasmic ribosomes was negligible when mitochondria were isolated in the presence of $\mathrm{Mg}^{2+}$. These findings are clearly at variance with the results we have presented and do not appear to be consistent with the findings of other authors $[14-16,19,25]$.

In interpreting the results of ribosomal protein analysis presented by van den Bogert and de Vries in relation to our results, several points should be considered. The differences reported for the protein patterns obtained with 80-S and 73-S ribosomes from mitochondrial preparations are substantial. Accordingly, the 73-S ribosomes cannot be simply derived from 80-S ribosomes by loss of some proteins. The authors have offered the interpretation that proteins selectively occurring in 80-S and 73-S ribosomes are generated by proteolytic degradation or modification. If such artifactual reactions do actually occur, it cannot be excluded that cytoplasmic ribosomes which contaminate mitochondria are altered in their ribosomal proteins. In addition, according to the isolation scheme of the authors [6], ribosomes adhering to mitochondria are treated quite differently to cytoplasmic ribosomes, which are directly isolated from the cell homogenate. The first type of cytoplasmic ribosomes is exposed to heparin and to $0.1 \mathrm{mM}$ EDTA in contrast to the second type. In our view it 
cannot be excluded that in this way alterations of ribosomal proteins and of protein composition occur which make it difficult to detect cytoplasmic ribosomal proteins in electrophoretic pattern of ribosomal proteins from $\mathrm{Mg}^{2+}$. isolated mitochondria, i.e. mixtures of mitochondrial and cytoplasmic ribosomes.

The effect of heparin on ribosomes isolated from different mitochondrial preparations

In gradients shown in Fig. 1 and Fig. 4 the ribosomes were isolated mainly as monosomes with relatively few polysomes. The addition of heparin is widely used as a means of inhibiting nucleases in the isolation of polysomes. We isolated SAMT- and SET-ribosomes in the presence of heparin. The gradient

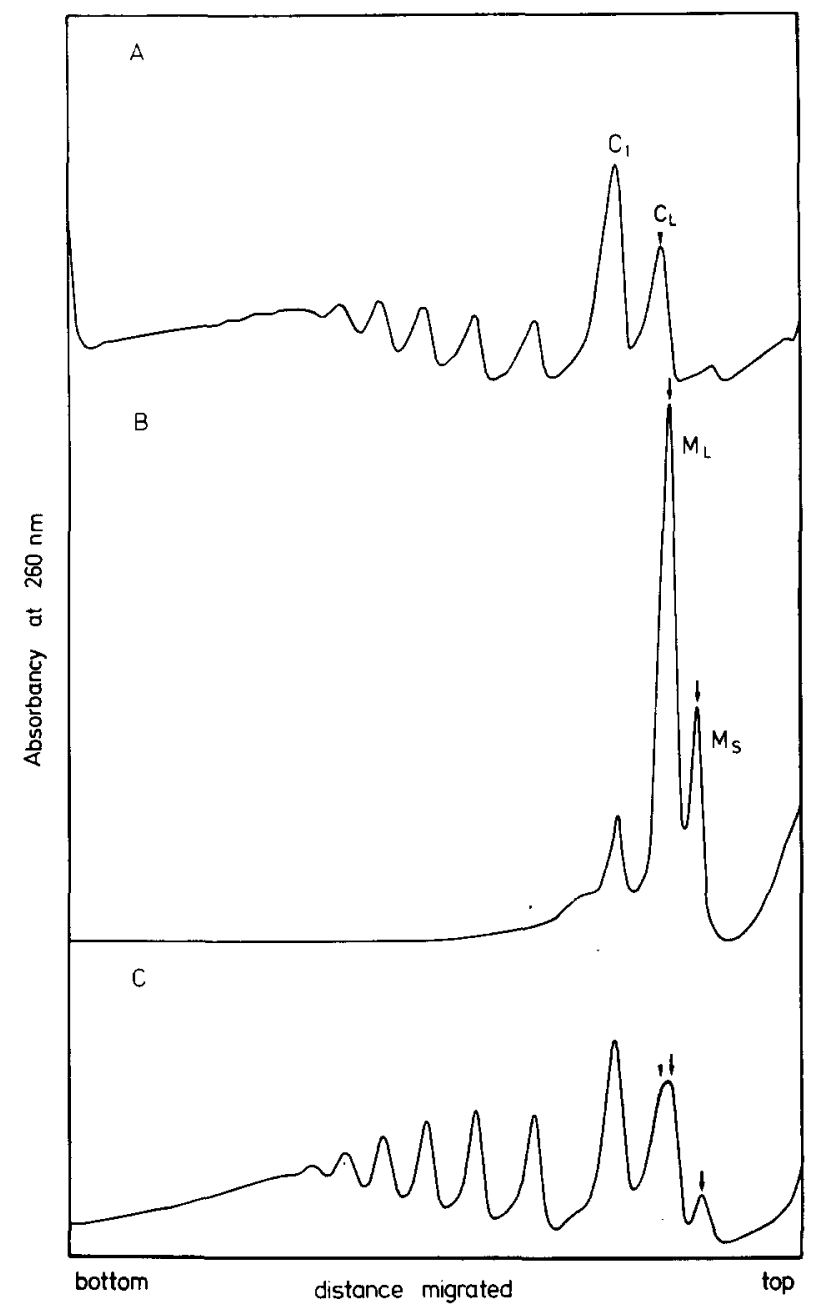

Fig. 5. Density gradient profiles of ribosomes isolated in the presence of heparin $(0.5 \mathrm{mg} / \mathrm{ml}$ isolation medium). A, cy toplasmic ribosomes: $\mathrm{B}$, ribosomes from $\mathrm{SE}_{1.0} \mathrm{~T}$-isolated mitochondria; $\mathrm{C}$, ribosomes from SAMT-isolated mitochondria. $C_{1}$, cytoplasmic monomer; $C_{L}$, cytoplasmic large subunit; $M_{L}, M_{S}, m_{i t o-}$ chondrial large and small subunits, respectively. 
profiles are shown in Figs. 5B and 5C. Included as a control are cytoplasmic ribosomes, prepared in the presence of heparin (Fig. $5 \mathrm{~A}$ ).

Cytoplasmic polyribosomes are effectively protected against breakdown or dissociation as evidenced by the relatively small amount of 79-S monomers and subunits relative to polysomes appearing in heparin preparations (Fig. 5A). The effect of heparin on the ribosomes from $\mathrm{SE}_{1.0} \mathrm{~T}$-isolated mitochondria, however, is quite striking. The ribosomes are almost completely dissociated and mainly subunits are obtained (Fig. 5B). The effect of heparin on ribosomes from SAMT-isolated mitochondria is shown in Fig. 5C. Polysomes are well preserved but the heparin causes disappearance of the $73 \mathrm{~S}$ component of the gradient. The 79-73 S double peak, shown in Fig. 1B, now becomes a single $79 \mathrm{~S}$ peak. Prolonged centrifugation of the gradients failed to reveal the presence of any $73 \mathrm{~S}$ component in the monomer peak of the heparin-isolated ribosomes from SAMT-isolated mitochondria. Concomitant with the disappearance of the $73 \mathrm{~S}$ peak is the appearance of 50-S and 37-S subunits. The broad subunit peak indicated by two arrows (Fig. 5C) can be shown to contain both cytoplasmic 60-S and mitochondrial 50-S subunits when the gradients are centrifuged for a longer time.

These findings lead us to conclude that heparin selectively dissociates the $73 \mathrm{~S}$ mitochondrial ribosome present in SAMT-isolated mitochondrial preparations. The resulting ribosomes contain only the surviving cytoplasmic ribosomes. The apparent homogeneity of 79-S ribosomes isolated from mitochondria in the presence of heparin appears not to be due to a stabilizing effect of heparin on mitochondrial ribosomes, as suggested by Datema et al. [5], but to the selective dissociation of mitochondrial ribosomes. The appearance of 50-S and 37-S subunits of SAMT-isolated mitochondrial ribosomes from SAMTisolated mitochondria in the presence of heparin is clearly shown in the gradients presented. These high levels of 50-S and 37-S subunits in robosomes from SAMT/heparin-isolated mitochondria are also been in the gradients presented by Datema et al. [5]. The effect of heparin on ribosomes from $\mathrm{SE}_{1.0} \mathrm{~T}$-isolated mitochondria has not been studied by these authors.

A keystone in the contention that $80-\mathrm{S}$ ribosomes are of mitochondrial origin is that the peptidyltransferase activity of ribosomes isolated from SAMT/ heparin-isolated mitochondria is inhibited by chloramphenicol but not by anisomycin. Since such preparations contain subunits of 73-S ribosomes, these 50-S subunits could confer sensitivity to chloramphenicol on the peptidyltransferase reaction [18].

\section{Ribosomal RNA from mitochondria isolated under different conditions}

RNA was extracted from mitochondria obtained by the various isolation procedures and subjected to gel electrophoresis. Electrophoretic profiles of the high molecular weight components are shown in Fig. 6. The EDTA-washed mitochondria ( $\mathrm{SE}_{1.0} \mathrm{~T}$, Fig. 6A) contain two large molecular weight RNAs with apparent molecular weights of $1.75 \cdot 10^{6}$ and $0.88 \cdot 10^{6}$. They occur in a ratio of $1.9 / 1$, which is almost the theoretical value of 2.0/1. A quite small amount of DNA is found near the start of the gel and constantly shows a double peak. The RNA profile from SAMT-isolated preparations (Fig. 6B) is quite different to the $\mathrm{SE}_{1.0} \mathrm{~T}$ profile and consists of four large molecular weight species with 


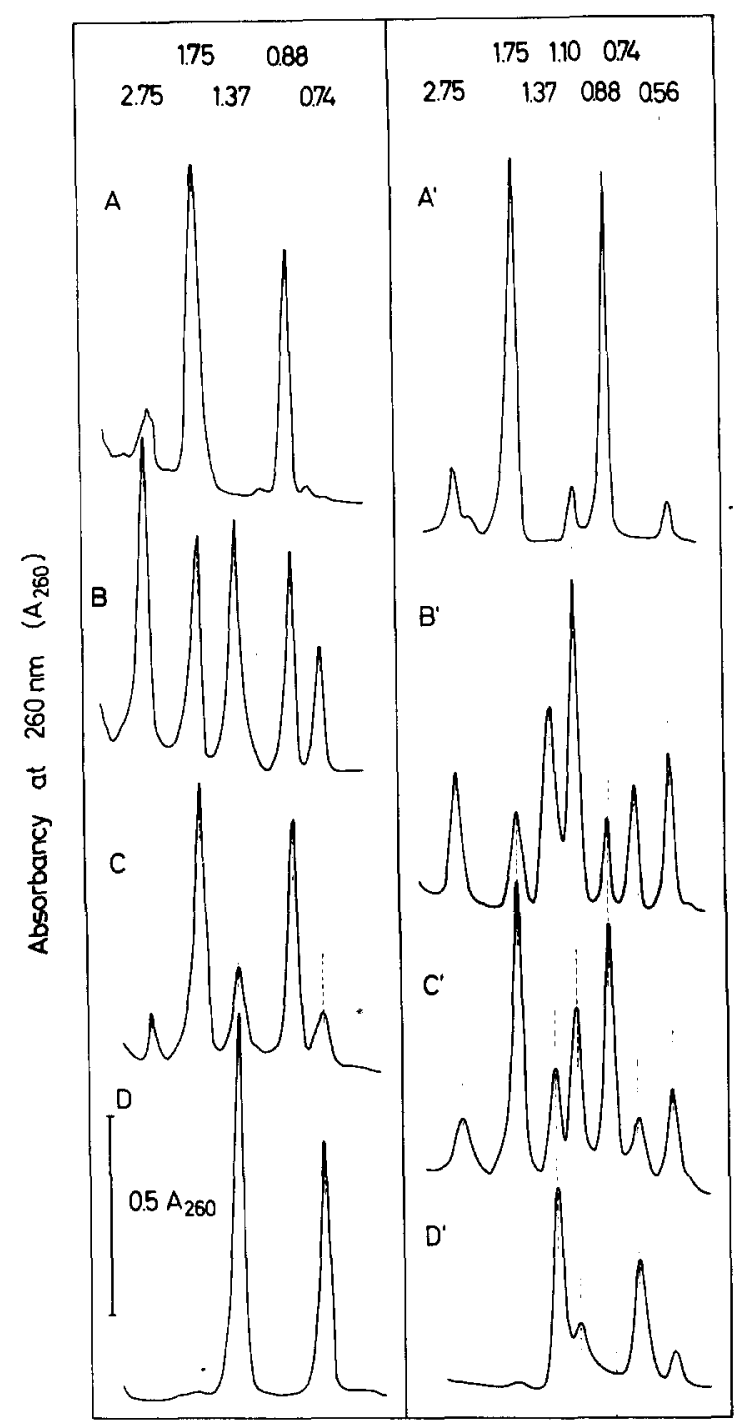

Fig. 6. Polyacrylamide gel electrophoresis of high molecular weight RNA extracted from mitochondria and from cytoplasm. A, SE 1.0 T-isolated mitochondria; B, SAMT-isolated mitochondria; C. SME-isolated mitochondria; D, total cytoplasmic RNA. Apparent molecular weights were determined by coelectrophoresis with $E$, coli ribosomal $R N A$ (cf, right column, $A^{\prime}, B^{\prime}, C^{\prime}, D^{\prime}$ ). The gels were run at $25-30^{\circ} \mathrm{C}$. Apparent molecular weights $\times 10^{-6}$ are shown at the top of the figure.

apparent molecular weight species with apparent molecular weights of $1.75 \cdot 10^{6}$, $1.37 \cdot 10^{6}, 0.88 \cdot 10^{6}$ and $0.74 \cdot 10^{6}$. The $1.75 \cdot 10^{6}$ and $0.88 \cdot 10^{6}$ dalton peaks are identical to the high molecular weight species found in $\mathrm{SE}_{1.0} \mathrm{~T}$-isolated mitochondria while the $1.37 \cdot 10^{6}$ and $0.74 \cdot 10^{6}$ dalton peaks are identical to the cytoplasmic ribosomal RNAs. This is obvious from a comparison with Fig. 6D in which cytoplasmic RNA is electrophoresed. The large size of the DNA peak in SAMT-isolated mitochondria (Fig. 6B) could correspond to the chromatin-like material frequently observed in electron micrographs (cf. Fig. 2B). A 
procedure intermediate between the $\mathrm{SE}_{1.0} \mathrm{~T}$ - and $\mathrm{SAMT}$-isolated preparations, viz. isolation in the presence of $\mathrm{Mg}^{2+}$ plus two washings with $0.1 \mathrm{mM}$ EDTA (SME, cf. Table I), results in gel profiles which show four high molecular weight species (Fig. 6C). However, in this instance the $1.75 \cdot 10^{6}$ and $0.88 \cdot 10^{6}$ dalton species are the major components, while the appearance of the cytoplasmic ribosomal RNA components is much reduced. The yield of total nucleic acid from SAMT-isolated mitochondria was consistently two to three times higher than from SET-isolated mitochondria. This can be attributed to the presence of large amounts of DNA and contaminating cytoplasmic RNA.

The high apparent molecular weight of the mitochondrial ribosomal RNA is due to the fact that the gels were run at $25^{\circ} \mathrm{C}$, a procedure which enhances the separation of the mitochondrial and cytoplasmic ribosomal RNAs. At this temperature partial unfolding of the mitochondrial ribosomal RNAs occurs [24]. When SAMT-preparations are run at $4^{\circ} \mathrm{C}$, the $1.75 \cdot 10^{6}$ dalton component runs considerably faster, and cannot be separated from the large component of the cytoplasmic ribosomal RNA, even after $4.5 \mathrm{~h}$ running time. The smaller components do separate but not completely under these condition [10].

It is concluded from these observations that mitochondria prepared in the presence of $10 \mathrm{mM} \mathrm{Mg}^{2+}$ contain not only mitochondrial RNA but also large amounts of cytoplasmic RNA and nuclear DNA.

We find ourselves in disagreement with Datema et al. [5] on the RNA composition of ribosomes in SAMT-isolated mitochondria. These authors have reported the occurrence of only one population of ribosomal RNAs. This is in sharp contrast to our findings and to those described by Lizardi and Luck [19], who have drawn the same conclusion as we. We cannot explain the homogeneity of the gel profiles presented by Datema et al. [5]. We can only suggest that their electrophoresis did not allow a clear resolution of all components present. Unfortunately these authors have not carried out coelectrophoresis of RNA from cytoplasmic and mitochondrial ribosomes to establish the resolving power of their electrophoresis.

A further controversy concerning Neurospora mitochondria is whether the mitochondrial ribosomes contain a $5 \mathrm{~S}$ RNA or not. Lizardi and Luck [19] have failed to detect a mitochondrial $5 \mathrm{~S}$ RNA in $N$. crassa. On the other hand, it was suggested that EDTA washing of mitochondria causes the loss of $5 \mathrm{~S}$ RNA from mitochondrial ribosomes [6,7]. We analysed total RNA extracts and extracts of purified ribosomes for the presence of $5 \mathrm{~S}$ RNA. The RNAs were separated on $7.5 \%$ gels and extracts were heated and cooled to reveal the presence of 5.8 S RNA (Fig. 7). $5 \mathrm{~S}$ RNA was evident in extracts of SAMTisolated mitochondria (Fig. 7B) but never in $\mathrm{SE}_{1.0} \mathrm{~T}$-isolated mitochondria (Fig. 7C). It was also present in extracts of cytoplasmic ribosomes (Fig. 7A).

Heating and cooling showed that extracts which contained $5 \mathrm{~S}$ RNA also contained 5.8 S RNA (cf. Figs. 7A and 7B). The latter RNA species is a characteristic constituent of cytoplasmic ribosomes but not of bacterial ribosomes $[20,21]$.

The simultaneous occurrence of $5 \mathrm{~S}$ and $5.8 \mathrm{~S}$ RNA in SAMT-isolated mitochondria leads us to the conclusion that $5 \mathrm{~S}$ RNA is not a constituent of mitochondrial ribosomes. Therefore, these RNA species are considered as indicative of the presence of cytoplasmic ribosomes. 


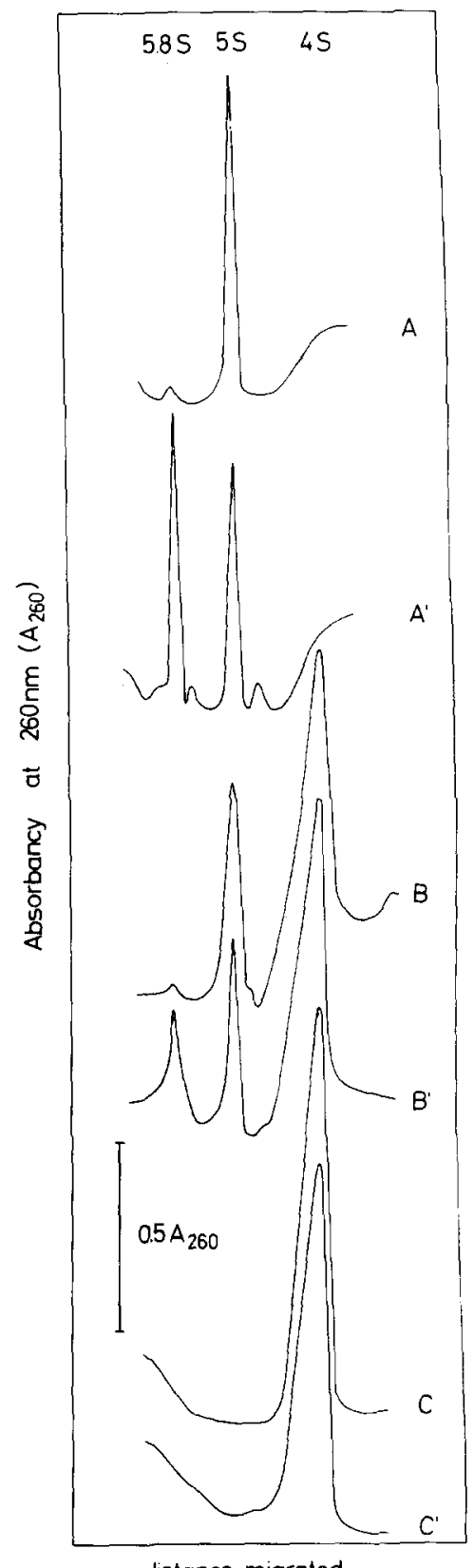

distance migrated

Fig. 7. Polyacrylamide gel electrophoresis of low molecular weight RNA extracted from cytoplasm and mitochondria under different conditions. A, cytoplasmic ribosomal RNA; B, SAMT-isolated mitochondria; $C, S E_{1,0} T^{-i s o l a t e d ~ m i t o c h o n d r i a . ~} A^{\prime}, B^{\prime}$ and $C^{\prime}$ same as $A, B$ and $C$, respectively, with exception that the RNA extracts were heated and cooled prior to electrophoresis.

\section{Conclusions}

The findings presented in this report show the presence of 79-S monosomes in preparations of ribosomes from mitochondria isolated in the presence of $\mathrm{Mg}^{2+}$. By a number of criteria these ribosomes were identified as cytoplasmic 
ribosomes. A mitochondrial ribosome with an $S$ value of $80 \mathrm{~S}$ could not be detected. The mitochondrial ribosome was found to be $73 \mathrm{~S}$ whether mitochondria were isolated in the presence of EDTA or $\mathrm{Mg}^{2+}$. This is in accordance with Küntzel and Noll's [4] first report on isolation of ribosomes from $N$. crassa, in which mitochondria were isolated and carefully purified in the presence of $\mathrm{Mg}^{2+}$. The use of heparin for the isolation may be misleading if its specific dissociation effect on mitochondrial 73-S ribosomes is neglected and may easily lead to the interpretation that the remaining cytoplasmic ribosomes are stabilized mitochondrial ribosomes.

The results lend strong support to the conclusion that the 73-S ribosomes are the original mitochondrial ribosomes and do not arise from an artifact. Unfortunately, only very few functional criteria for the integrity of mitochondrial ribosomes are available. Ribosomes in EDTA-isolated mitochondria were shown to be able to synthesize the same polypeptides as in vivo. Particularly, they synthesize subunits of cytochrome oxidase immunoprecipitable with antibodies to the whole enzyme [22]. It is difficult to visualize how a ribosome; which according to the proposal of Kroon et al. [7] has lost $5 \mathrm{~S}$ RNA and several proteins, has retained this functional activity.

\section{Acknowledgements}

This work was supported by the Deutsche Forschungsgemeinschaft, Schwerpunktsprogramm "Biochemie der Morphogenese". Dr. M.A. Harmey wishes to thank CIBA GEIGY for a visiting fellowship.

\section{References}

1 O'Brien, T.W. and Matthews, D.E. (1976) in Handbook of Genetics (King, R.C., ed.), Vol. 5, pp. 535-580, Plenum Publishing Corp., New York

2 Avadhani, N.G., Lewis, F.S. and Rutman, R.J. (1975) Sub.-Cell. Biochem. 4, 93-145

3 Neupert, W. (1977) in Horizons in Biochemistry and Biophysics (Quagliariello, E., Palmieri, F. and Singer, T.P., eds.), Vol. 3, pp. 257-296 Addison-Wesley Publ. Co., Reading, Mass.

4 Küntzel, H. and Noll, H. (1967) Nature 215, 1340-1345

5 Datema, R., Agsteribbe, E. and Kroon, A.M. (1974) Biochim. Biophys. Acta 335, $386-395$

6 van den Bogert, C. and de Vries, H. (1976) Biochim. Biophys. Acta 442, 227-238

7 Kroon, A.M., Terpesta, P., Holtrop, M., de Vries, H., van den Bogert, C., de Jonge, J. and Agsteribbe, E. (1976) in Genetics and Biogenesis of Chloroplasts and Mitochondria (Bücher, Th., Neupert, W., Sebald, W. and Werner, S., eds.), pp. 685-696, North-Holland, Amsterdam

8 Vogel, H.J. (1956) Microbiol. Genet. Bull. 13, 42-48

9 Weiss, H., vaon Jagow, G., Klingenberg, M. and Bücher, Th. (1970) Eur. J. Biochem. 10, 75-82

10 Neupert, W., Sebald, W., Schwab, A., Pfaller, A. and Bücher, Th. (1969) Eur. J. Biochem. 10, 585588

11 Hallermayer, G. and Neupert, W. (1974) FEBS Lett. 41, 264-268

12 Leaver, C.J. and Ingle, J. (1971) Biochem. J. 123, 235--243

13 Loening, U.E. (1969) Biochem. J. 113,131-138

14 Palmiter, R.D. (1974) Biochemistry 13, 3606-3615

15 Adelmann, M.R., Blobel, G. and Sabatini, D.D. (1973) J. Cell Biol. 56, 191-205

16 Kellems, R.E. and Butow, R.A. (1972) J. Biol. Chem. 247, 8043-8050

17 Alberghina, F.A.M. and Suskind, S.R. (1967) J. Bacteriol. 94, 630-649

18 Monro, R.E., Cerna, J. and Marcker, K.A. (1968) Proc. Natl. Acad. Sci. U.S. 61, $1042-1049$

19 Lizardi, P.M. and Luck, D.J.L. (1971) Nat. New Biol. 229, 140-142

20 Pene, J.J., Knight, E. and Darnell, J.E. (1968) J. Mol. Biol. 33, 609-623

21 Leaver, C.J. and Harmey, M.A. (1976) Biochem. J. 157, 275-277

22 von Rücker, A., Neupert, W, and Werner, S. (1976) in Genetics, Biogenesis and Bioenergetics of Mitochondria (Bandlow, W., Schweyen, R.J., Thomas, D.Y., Wolf, K. and Kandewitz, F., eds.), pp. 259-267, Walter de Gruyter, Berlin

23 Taylor, M.M. and Storck, R. (1964) Proc. Nati. Acad. Sci. U.S. 52, 958-965

24 Grivell, L.A., Reijnders, L. and Borst, P. (1971) Eur. J. Biochem. 19, 64-72

25 Schmitt, H, (1970) Eur. J. Biochem. 17, 278-283 